Mathematical Modelling and Analysis

Volume 16 Number 1, March 2011, 153-172

Doi:10.3846/13926292.2011.564771

(C) Vilnius Gediminas Technical University, 2011
www.tandf.co.uk/journals/TMMA

Publisher: Taylor\&Francis and VGTU

Online ISSN: 1648-3510

Print ISSN: 1392-6292

\title{
Product Integration for Weakly Singular Integro-Differential Equations*
}

\author{
A. Pedas and E. Tamme \\ Institute of Mathematics, University of Tartu \\ Liivi 2, 50409 Tartu, Estonia \\ E-mail: arvet.pedas@ut.ee \\ E-mail(corresp.): enn.tamme@ut.ee
}

Received September 18, 2010; revised February 12, 2011; published online March 1, 2011

\begin{abstract}
On the basis of product integration techniques a discrete version of a piecewise polynomial collocation method for the numerical solution of initial or boundary value problems of linear Fredholm integro-differential equations with weakly singular kernels is constructed. Using an integral equation reformulation and special graded grids, optimal global convergence estimates are derived. For special values of parameters an improvement of the convergence rate of elaborated numerical schemes is established. Presented numerical examples display that theoretical results are in good accordance with actual convergence rates of proposed algorithms.
\end{abstract}

Keywords: weakly singular integro-differential equation, collocation method, product integration, graded grid.

AMS Subject Classification: 65R20; 45J05.

\section{Introduction}

Let $\mathbb{R}=(-\infty, \infty)$ and $\mathbb{N}=\{1,2, \ldots\}$. In the present paper we study the convergence behaviour of a discrete version of a collocation method for the numerical solution of initial or boundary value problems of the form

$$
\begin{aligned}
& u^{(n)}(t)=\sum_{i=0}^{n_{1}} a_{i}(t) u^{(i)}(t)+\sum_{i=0}^{n_{0}} \int_{0}^{b} g_{i}(t, s) K_{i}(t, s) u^{(i)}(s) d s+f(t), \\
& \sum_{i=0}^{n-1}\left[\alpha_{i j} u^{(i)}(0)+\beta_{i j} u^{(i)}(b)\right]=0, \quad j=1, \ldots, n,
\end{aligned}
$$

where $0 \leq t \leq b, n \in \mathbb{N}, 0 \leq n_{0} \leq n, 0 \leq n_{1} \leq n-1, \alpha_{i j}, \beta_{i j} \in \mathbb{R}(i=$ $0, \ldots, n-1, j=1, \ldots, n)$ and $f, a_{i}:[0, b] \rightarrow \mathbb{R}\left(i=0, \ldots, n_{1}\right)$ are some continuous functions. We assume that $K_{i} \in C^{q}([0, b] \times[0, b]), g_{i} \in W^{q, \nu}(\Delta)$

* The work was supported by Estonian Science Foundation Grant No. 7353. 
$\left(i=0, \ldots, n_{0}\right)$ with $q \in \mathbb{N}$ and $-\infty<\nu<1$, and the integration of $g_{i}(t, s) \varphi(s)$ can be carried out analytically (exactly) for any polynomial $\varphi(s)$.

For given $q \in \mathbb{N}$ and $-\infty<\nu<1$, by $W^{q, \nu}(\Delta)$,

$$
\Delta=\{(t, s): 0 \leq t \leq b, 0 \leq s \leq b, t \neq s\}
$$

is denoted the set of $q$ times continuously differentiable functions $g: \Delta \rightarrow \mathbb{R}$ satisfying for all $(t, s) \in \Delta$ and all non-negative integers $i$ and $j$ such that $i+j \leq q$ the condition

$$
\left|\left(\frac{\partial}{\partial t}\right)^{i}\left(\frac{\partial}{\partial t}+\frac{\partial}{\partial s}\right)^{j} g(t, s)\right| \leq c \begin{cases}1 & \text { if } \nu+i<0 \\ 1+|\log | t-s|| & \text { if } \nu+i=0 \\ |t-s|^{-\nu-i} & \text { if } \nu+i>0\end{cases}
$$

where $c=c(g)$ is a positive constant. Note that $g_{i} \in W^{q, \nu}(\Delta)\left(i=0, \ldots, n_{0}\right)$ with $0<\nu<1$ and arbitrary $q \in \mathbb{N}$ if $g_{i}(t, s)=1$ or $g_{i}(t, s)=\log ^{k_{i}}|t-s|$ $\left(k_{i} \in \mathbb{N}\right)$ or $g_{i}(t, s)=|t-s|^{-\alpha_{i}}$ where $\alpha_{i} \leq \nu$. We have $g_{i} \in W^{q, 0}(\Delta)(i=$ $\left.0, \ldots, n_{0}\right)$ with arbitrary $q \in \mathbb{N}$ if $g_{i}(t, s)=\log |t-s|$ or $g_{i}(t, s)=|t-s|^{-\alpha_{i}}$ where $\alpha_{i} \leq 0$.

Since the kernels $g_{i}(t, s) K_{i}(t, s)\left(i=0, \ldots, n_{0}\right)$ of equation (1.1) may have a weak singularity on the diagonal $t=s$, the derivatives of the resulting solution $u$ of order greater than $n$ are typically unbounded near the endpoints of the interval $[0, b]$ (see Lemma 2 in Section 2). In collocation methods the possible singular behaviour of the solution of (1.1), (1.2) can be taken into account by using polynomial splines [10], see also [4, 9]. However, the convergence results established in these works are derived under the assumption that the integrals occurring in the collocation equation can be evaluated analytically (exactly). Since this is rarely possible in concrete applications, there arises the question how to approximate these integrals, and it is of interest to derive error estimates for the approximate solutions. In [6] this problem in the case of special quadrature formulas is studied.

In the present paper, using an integral equation reformulation of problem (1.1), (1.2), we first discretize the corresponding integral equation by quadrature formulas based on product integration (see, for example, $[2,3]$ ) and then apply a piecewise polynomial collocation method on special graded grids reflecting the singular behaviour of the exact solution. With this approach we approximate smooth parts of the corresponding integrands by piecewise polynomial interpolation and then integrate exactly the remaining (more singular) parts of these integrands (see Section 4). Often such method is called a discrete collocation method [2]. Similar approach for solving integral equations has been used in $[1,2,3,7,8,14,15]$. In [12] with the help of similar ideas a fully discrete version of the Galerkin method is constructed.

The purpose of the present paper is to show that such discrete collocation method is convergent for a sufficient wide class of weakly singular integrodifferential equations and to study the attainable order of global convergence of this method. The main results of the paper extend and refine the corresponding results of $[9,10]$ and are formulated in Theorems 1 and 2. In Section 7 these results are verified by some numerical examples. 


\section{Smoothness of the Solution}

In order to formulate a smoothness result about the solution of $(1.1),(1.2)$, we introduce a set of functions $C^{q, \nu}(0, b)$. For given $q \in \mathbb{N}$ and $-\infty<\nu<1$, by $C^{q, \nu}(0, b)$ we denote the set of continuous functions $u:[0, b] \rightarrow \mathbb{R}$ which are $q$ times continuously differentiable in $(0, b)$ and such that for all $t \in(0, b)$ and $i=0, \ldots, q$ the following estimate holds:

$$
\left|u^{(i)}(t)\right| \leq c \begin{cases}1 & \text { if } i<1-\nu \\ 1+|\log \varrho(t)| & \text { if } i=1-\nu \\ \varrho(t)^{1-\nu-i} & \text { if } i>1-\nu\end{cases}
$$

Here $c=c(u)$ is a positive constant and $\varrho(t)=\min \{t, b-t\}, 0<t<b$, is the distance from $t \in(0, b)$ to the boundary of the interval $(0, b)$. Equipped with the norm

$$
\|u\|_{q, \nu}=\max _{0 \leq t \leq b}|u(t)|+\sum_{i=1}^{q} \sup _{0<t<b}\left(w_{i+\nu-1}(t)\left|u^{(i)}(t)\right|\right), \quad u \in C^{q, \nu}(0, b),
$$

$C^{q, \nu}(0, b)$ is a Banach space. Here

$$
w_{\lambda}(t)= \begin{cases}1 & \text { for } \lambda<0 \\ (1+|\log \varrho(t)|)^{-1} & \text { for } \lambda=0 \\ \varrho(t)^{\lambda} & \text { for } \lambda>0\end{cases}
$$

with $t \in(0, b)$. Clearly, $C^{q}[0, b] \subset C^{q, \nu}(0, b) \subset C[0, b]$ with $-\infty<\nu<1$ and arbitrary $q \in \mathbb{N}$. Note that the functions in the form

$$
u(t)=b_{1}(t) t^{1-\alpha}+b_{2}(t)(b-t)^{1-\beta}+b_{3}(t), \quad 0<t<b,
$$

are included in $C^{q, \nu}(0, b)(q \in \mathbb{N}, \nu<1)$ if $\alpha \leq \nu, \beta \leq \nu$ and $b_{j} \in C^{q}[0, b]$, $j=1,2,3$.

In the sequel we use a reformulation of problem (1.1), (1.2) based on introducing a new unknown function $v=u^{(n)}$. If from all polynomials $u$ of degree $n-1$ only $u=0$ satisfies the conditions (1.2), then the equation

$$
u^{(n)}(t)=v(t), \quad t \in[0, b], v \in L^{\infty}(0, b),
$$

with boundary conditions (1.2), has a unique solution

$$
u(t)=\int_{0}^{b} G(t, s) v(s) d s, \quad t \in[0, b]
$$

where $G(t, s)$ is the Green function of problem (2.2), (1.2). The derivatives of the function $u$ given by $(2.3)$ can be expressed in the form

$$
u^{(i)}(t)=\left(J_{i} v\right)(t), \quad t \in[0, b], i=0, \ldots, n-1,
$$

where

$$
\left(J_{i} v\right)(t)=\int_{0}^{b} \frac{\partial^{i} G(t, s)}{\partial t^{i}} v(s) d s, \quad t \in[0, b], i=0, \ldots, n-1 .
$$


We set $J_{n} v=v$, i.e. $J_{n}=I$ is the identity operator. Since the general solution of equation $u^{(n)}(t)=0$ is an arbitrary polynomial of degree $n-1$, the Green function $G(t, s)$ for $(2.2),(1.2)$ can be expressed both for $t<s$ and for $t>s$ as the polynomial at most of degree $n-1$ with respect to $t$ and $s$. Moreover, $\partial^{i} G(t, s) / \partial t^{i}, i=0, \ldots, n-2$, the derivatives of $G(t, s)$ with respect to $t$ up to the order $n-2$, are continuous on $\bar{\Delta}=[0, b] \times[0, b]$. Also $\partial^{n-1} G(t, s) / \partial t^{n-1}$ is continuous and bounded in the region $\Delta$, but it has a discontinuity at $t=s$.

Note that in [6] a different formula for $J_{i}$ is derived. The operators $J_{i}$, $i=0, \ldots, n-1$, have the following properties proved in [10].

Lemma 1. Assume that from all polynomials $u$ of degree $n-1$ only $u=0$ satisfies the conditions (1.2). Then $J_{i}, i \in\{0, \ldots, n-1\}$, defined by $(2.5)$ is linear and compact as an operator from $L^{\infty}(0, b)$ into $C[0, b]$. Moreover, $J_{i}$ is a bounded operator from $C^{q, \nu}(0, b)$ into $C^{q+n-i, \nu-n+i}(0, b)$ for every $q \in \mathbb{N}$ and $\nu<1$.

Using $u^{(n)}=v$ and (2.4), problem (1.1), (1.2) may be rewritten as a linear integral equation with respect to $v$ :

$$
v=T v+f
$$

where

$$
\begin{gathered}
T=\sum_{i=0}^{n_{1}} A_{i} J_{i}+\sum_{i=0}^{n_{0}} \tilde{T}_{i} J_{i} \\
\left(A_{i} z\right)(t)=a_{i}(t) z(t), \quad\left(\tilde{T}_{i} z\right)(t)=\int_{0}^{b} g_{i}(t, s) K_{i}(t, s) z(s) d s, \quad t \in[0, b] .
\end{gathered}
$$

Equation (2.6) is equivalent to the problem (1.1), (1.2) in the following sense: if $u \in C^{n}[0, b]$ is a solution to (1.1), (1.2) then $v=u^{(n)}$ is a solution to (2.6); conversely, if $v \in C[0, b]$ is a solution to (2.6) then $u=J_{0} v$ is a solution to $(1.1),(1.2)$.

The existence and regularity of the solution of problem (1.1), (1.2) can be characterized by the following lemma.

Lemma 2. Assume that $f \in C^{q, \nu}(0, b), a_{i} \in C^{q, \nu}(0, b), i=0, \ldots, n_{1}, g_{i} \in$ $W^{q, \nu}(\Delta), K_{i} \in C^{q}([0, b] \times[0, b]), i=0, \ldots, n_{0}, 0 \leq n_{0} \leq n, 0 \leq n_{1} \leq n-1$, $q, n \in \mathbb{N},-\infty<\nu<1$. Moreover, assume that the problem (1.1), (1.2) with $f=0$ has only the trivial solution $u=0$ and from all polynomials $u$ of degree $n-1$ only $u=0$ satisfies the conditions (1.2). Then problem (1.1), (1.2) possesses a unique solution $u \in C^{q+n, \nu-n}(0, b)$ and for its derivatives $u^{\prime}, u^{\prime \prime}, \ldots, u^{(n)}$ we have that $u^{(i)} \in C^{q+n-i, \nu-n+i}(0, b), i=1, \ldots, n$.

Proof. Let us consider the equation (2.6) which is equivalent to the problem (1.1), (1.2). Since $a_{i} \in C^{q, \nu}(0, b), A_{i}\left(i=0, \ldots, n_{1}\right)$ are linear and bounded as operators from $C[0, b]$ into $C[0, b]$ and also from $C^{q, \nu}(0, b)$ into $C^{q, \nu}(0, b)$ (see [10]). It follows from $[13]$ that $J_{i}(i=0, \ldots, n-1)$ and $\tilde{T}_{i}\left(i=0, \ldots, n_{0}\right)$ and consequently also $T$ (defined by $(2.7)$ ) are linear and compact as operators from $C[0, b]$ into $C[0, b]$ and from $C^{q, \nu}(0, b)$ into $C^{q, \nu}(0, b)$. Since the problem 
(1.1), (1.2) with $f=0$ has only the trivial solution, the homogeneous equation $v=T v$ possesses in $C[0, b] \supset C^{q, \nu}(0, b)$ only the trivial solution $v=0$. This together with the Fredholm alternative theorem yields that equation (2.6) is uniquely solvable in $C^{q, \nu}(0, b)$ and therefore, its solution $v$ belongs to $C^{q, \nu}(0, b)$. With the help of Lemma 1 we now obtain that problem (1.1), (1.2) has a unique solution $u=J_{0} v \in C^{q+n, \nu-n}(0, b)$ and its derivatives $u^{(i)}=J_{i} v \in$ $C^{q+n-i, \nu-n+i}(0, b), i=1, \ldots, n$.

Remark 1. For $0 \leq n_{0} \leq n-1$ Lemma 2 follows also from Theorem 2.1 of [10].

\section{Piecewise Polynomial Interpolation}

We will seek an approximate solution for problem (1.1), (1.2) in the form of a piecewise polynomial function on a nonuniform grid reflecting the possible singular behaviour of the exact solution of (1.1), (1.2). For given $N \in \mathbb{N}$, let

$$
\Pi_{N}=\left\{t_{0}, \ldots, t_{2 N}: 0=t_{0}<t_{1}<\ldots<t_{2 N}=b\right\}
$$

be a partition (a graded grid) of the interval $[0, b]$ with the grid points

$$
t_{j}=\frac{b}{2}\left(\frac{j}{N}\right)^{r}, \quad j=0,1, \ldots, N, \quad t_{N+j}=b-t_{N-j}, \quad j=1, \ldots, N,
$$

where the grading exponent $r \in \mathbb{R}, r \geq 1$. If $r=1$, then the grid points (3.1) are distributed uniformly; for $r>1$ the points (3.1) are more densely clustered near the endpoints of the interval $[0, b]$. Let $S_{m}^{(-1)}\left(\Pi_{N}\right)$ and $C^{(-1)}\left(\Pi_{N}\right)$ be the following spaces of piecewise continuous functions on $[0, b]$ :

$$
\begin{aligned}
& S_{m}^{(-1)}\left(\Pi_{N}\right)=\left\{v:\left.v\right|_{\left[t_{j-1}, t_{j}\right]} \in \pi_{m}, j=1, \ldots, 2 N\right\}, \quad m \geq 0, \\
& C^{(-1)}\left(\Pi_{N}\right)=\left\{v:\left.v\right|_{\left[t_{j-1}, t_{j}\right]} \in C\left[t_{j-1}, t_{j}\right], j=1, \ldots, 2 N\right\} .
\end{aligned}
$$

Here $\left.v\right|_{\left[t_{j-1}, t_{j}\right]}$ is the restriction of $v$ onto the subinterval $\left[t_{j-1}, t_{j}\right], j=1, \ldots, 2 N$, and $\pi_{m}$ denotes the set of polynomials of degree not exceeding $m$. We accept that at the interior points $t_{1}, \ldots, t_{2 N-1}$ of the grid $\Pi_{N}$ the elements of $S_{m}^{(-1)}\left(\Pi_{N}\right)$ and $C^{(-1)}\left(\Pi_{N}\right)$ may have two values. Equipped with the norm

$$
\|v\|_{\infty}=\max _{j=1, \ldots, 2 N} \sup _{t_{j-1}<t<t_{j}}|v(t)|, \quad v \in C^{(-1)}\left(\Pi_{N}\right),
$$

$C^{(-1)}\left(\Pi_{N}\right)$ is a Banach space. Clearly, $C[0, b] \subset C^{(-1)}\left(\Pi_{N}\right) \subset L^{\infty}(0, b)$.

In every subinterval $\left[t_{j-1}, t_{j}\right], j=1, \ldots, 2 N$, we introduce $m \geq 1$ collocation points

$$
t_{j k}=t_{j-1}+\eta_{k}\left(t_{j}-t_{j-1}\right), \quad k=1, \ldots, m,
$$

and $m_{1} \geq 1$ interpolation nodes

$$
s_{j k}=t_{j-1}+\xi_{k}\left(t_{j}-t_{j-1}\right), \quad k=1, \ldots, m_{1},
$$

where $\eta_{1}, \ldots, \eta_{m}$ and $\xi_{1}, \ldots, \xi_{m_{1}}$ are two fixed systems of parameters which do not depend on $j$ and $N$ and satisfy the conditions

$$
0 \leq \eta_{1}<\ldots<\eta_{m} \leq 1, \quad 0 \leq \xi_{1}<\ldots<\xi_{m_{1}} \leq 1
$$


We define two interpolation operators $\mathcal{P}_{N}: C^{(-1)}\left(\Pi_{N}\right) \rightarrow S_{m-1}^{(-1)}\left(\Pi_{N}\right)$ and $\mathcal{Q}_{N}: C^{(-1)}\left(\Pi_{N}\right) \rightarrow S_{m_{1}-1}^{(-1)}\left(\Pi_{N}\right)$ requiring that

$$
\begin{aligned}
& \mathcal{P}_{N} v \in S_{m-1}^{(-1)}\left(\Pi_{N}\right),\left(\mathcal{P}_{N} v\right)\left(t_{j k}\right)=v\left(t_{j k}\right), k=1, \ldots, m, j=1, \ldots, 2 N \\
& \mathcal{Q}_{N} v \in S_{m_{1}-1}^{(-1)}\left(\Pi_{N}\right),\left(\mathcal{Q}_{N} v\right)\left(s_{j k}\right)=v\left(s_{j k}\right), k=1, \ldots, m_{1}, j=1, \ldots, 2 N
\end{aligned}
$$

for any piecewise continuous function $v \in C^{(-1)}\left(\Pi_{N}\right)$. If $\eta_{1}=0$ then by $\left(\mathcal{P}_{N} v\right)\left(t_{j 1}\right)$ and $v\left(t_{j 1}\right)$ we mean the right limits

$$
\left(\mathcal{P}_{N} v\right)\left(t_{j 1}\right)=\lim _{t \rightarrow t_{j 1}, t>t_{j 1}}\left(\mathcal{P}_{N} v\right)(t) \quad \text { and } \quad v\left(t_{j 1}\right)=\lim _{t \rightarrow t_{j 1}, t>t_{j 1}} v(t)
$$

respectively. If $\eta_{m}=1$ then $\left(\mathcal{P}_{N} v\right)\left(t_{j m}\right)$ and $v\left(t_{j m}\right)$ denote the left limits

$$
\left(\mathcal{P}_{N} v\right)\left(t_{j m}\right)=\lim _{t \rightarrow t_{j m}, t<t_{j m}}\left(\mathcal{P}_{N} v\right)(t) \quad \text { and } \quad v\left(t_{j m}\right)=\lim _{t \rightarrow t_{j m}, t<t_{j m}} v(t)
$$

respectively. In analogy with this in (3.5) at the endpoints of the interval $\left[t_{j-1}, t_{j}\right]$ we will use the right and left one-side limits of $\left(\mathcal{Q}_{N} v\right)(t), v(t)$ at $t=s_{j 1}=t_{j-1}$ (if $\left.\xi_{1}=0\right)$ and $t=s_{j m_{1}}=t_{j}$ (if $\left.\xi_{m_{1}}=1\right)$, respectively.

For such interpolation the following error estimates are valid (see [16]).

Lemma 3. Let $v \in C^{m, \nu}(0, b), m \in \mathbb{N},-\infty<\nu<1$, and let $\mathcal{P}_{N} v$ be defined by (3.4) where the nodes (3.2) with grid points (3.1) are used. Then the following estimates hold:

$$
\begin{aligned}
& \left\|v-\mathcal{P}_{N} v\right\|_{\infty} \leq c E_{N}(m, \nu, r)\|v\|_{m, \nu} \\
& \left\|v-\mathcal{P}_{N} v\right\|_{1} \leq c \Theta_{N}(m, \nu, r)\|v\|_{m, \nu} .
\end{aligned}
$$

Here $c$ does not depend on $N \in \mathbb{N}$ and $v \in C^{m, \nu}(0, b)$ and

$$
\begin{gathered}
\left\|v-\mathcal{P}_{N} v\right\|_{1}=\int_{0}^{b}\left|v(t)-\left(\mathcal{P}_{N} v\right)(t)\right| d t \\
E_{N}(m, \nu, r)= \begin{cases}N^{-r(1-\nu)} & \text { for } 1 \leq r<\frac{m}{1-\nu}, \\
N^{-m}(1+\log N) & \text { for } r=\frac{m}{1-\nu}=1, \\
N^{-m} & \text { for } r=\frac{m}{1-\nu}>1, \\
\Theta_{N}(m, \nu, r)= \begin{cases}N^{-r(2-\nu)} \\
N^{-m}(1+\log N)\end{cases} & \text { for } 1 \leq r<\frac{m}{1-\nu}, r \geq 1 ; \\
N^{-m} & \text { for } r>\frac{m}{2-\nu} \geq 1,\end{cases}
\end{gathered}
$$

Remark 2. It is easy to see that $\Theta_{N}(m, \nu, r) \leq E_{N}(m, \nu, r)$ for $N, m \in \mathbb{N}$, $-\infty<\nu<1$ and $r \geq 1$. 


\section{Discrete Collocation Method}

Using ideas of product integration we approximate the equation (2.6) by a sequence of equations

$$
v=T_{N} v+f, \quad N \in \mathbb{N},
$$

where

$$
\begin{aligned}
& T_{N}=\sum_{i=0}^{n_{1}} A_{i} J_{i}+\sum_{i=0}^{n_{0}} \tilde{T}_{i N} J_{i}, \\
& \left(\tilde{T}_{i N} z\right)(t)=\int_{0}^{b} g_{i}(t, s) \mathcal{Q}_{N}\left[K_{i}(t, \cdot) z(\cdot)\right](s) d s, \quad t \in[0, b] .
\end{aligned}
$$

Here the operator $\mathcal{Q}_{N}$ defined by (3.5) is applied to the product $K_{i}(t, s) z(s)$ as the function of $s$ treating $t$ as a parameter, and $J_{i}$ and $A_{i}$ are given by (2.5) and (2.8), respectively.

Further, we look for an approximation $v_{N}$ to the solution $v$ of equation (2.6) in $S_{m-1}^{(-1)}\left(\Pi_{N}\right), m, N \in \mathbb{N}$. We determine $v_{N} \in S_{m-1}^{(-1)}\left(\Pi_{N}\right)$ by the collocation conditions

$$
v_{N}\left(t_{j k}\right)=\left(T_{N} v_{N}\right)\left(t_{j k}\right)+f\left(t_{j k}\right), \quad k=1, \ldots, m, j=1, \ldots, 2 N .
$$

Here by $v_{N}\left(t_{j 1}\right)$ and $v_{N}\left(t_{j m}\right)$ are denoted the right and left limits of $v_{N}(t)$ at $t_{j 1}$ and $t_{j m}$, respectively. This method can be presented equivalently in the following form: find $v_{N}$ such that

$$
v_{N}=\mathcal{P}_{N} T_{N} v_{N}+\mathcal{P}_{N} f
$$

with $\mathcal{P}_{N}$, defined by (3.4). This method is often called as a discrete collocation method for solving (2.6) (cf. [2]). Let us present an algorithm for finding $v_{N}$. Using the Lagrange interpolation formula in every interval $\left[t_{j-1}, t_{j}\right]$, we get from (3.4) and (3.5) the following expressions for $\mathcal{P}_{N} v$ and $\mathcal{Q}_{N} v$ :

$$
\begin{aligned}
& \left(\mathcal{P}_{N} v\right)(t)=\sum_{p=1}^{2 N} \sum_{q=1}^{m} v\left(t_{p q}\right) \varphi_{p q}(t), \quad t \in[0, b], \\
& \left(\mathcal{Q}_{N} v\right)(t)=\sum_{p=1}^{2 N} \sum_{q=1}^{m_{1}} v\left(s_{p q}\right) \psi_{p q}(t), \quad t \in[0, b],
\end{aligned}
$$

where $\varphi_{p q}(t)=0$ and $\psi_{p q}(t)=0$ if $t \notin\left[t_{p-1}, t_{p}\right]$ and

$$
\begin{aligned}
& \varphi_{p q}(t)=\prod_{k=1, k \neq q}^{m} \frac{t-t_{p k}}{t_{p q}-t_{p k}} \quad \text { if } t \in\left[t_{p-1}, t_{p}\right], p=1, \ldots, 2 N, q=1, \ldots, m, \\
& \psi_{p q}(t)=\prod_{k=1, k \neq q}^{m_{1}} \frac{t-s_{p k}}{s_{p q}-s_{p k}} \quad \text { if } t \in\left[t_{p-1}, t_{p}\right], p=1, \ldots, 2 N, q=1, \ldots, m_{1} .
\end{aligned}
$$


For $\tilde{T}_{i N}$ defined by (4.2) we obtain with the help of (4.5) that

$$
\left(\tilde{T}_{i N} z\right)(t)=\sum_{\alpha=1}^{2 N} \sum_{\beta=1}^{m_{1}} w_{i \alpha \beta}(t) K_{i}\left(t, s_{\alpha \beta}\right) z\left(s_{\alpha \beta}\right), \quad t \in[0, b], i=0, \ldots, n_{0},
$$

where $w_{i \alpha \beta}(t)=\int_{t_{\alpha-1}}^{t_{\alpha}} g_{i}(t, s) \psi_{\alpha \beta}(s) d s$. We assume that the weights $w_{i \alpha \beta}(t)$ can be evaluated exactly. Searching the solution of (4.3) in the form

$$
v_{N}(t)=\sum_{p=1}^{2 N} \sum_{q=1}^{m} c_{p q} \varphi_{p q}(t), \quad t \in[0, b],
$$

we obtain the following linear system with respect to the coefficients $c_{p q}=$ $v_{N}\left(t_{p q}\right)$ :

$$
c_{j k}=\sum_{p=1}^{2 N} \sum_{q=1}^{m} a_{j k p q} c_{p q}+f\left(t_{j k}\right), \quad k=1, \ldots, m, j=1, \ldots, 2 N,
$$

where $a_{j k p q}=\left(T_{N} \varphi_{p q}\right)\left(t_{j k}\right)$.

Remark 3. If $\eta_{1}=0, \eta_{m}=1$ then $t_{j m}=t_{j+1,1}=t_{j}, j=1, \ldots, 2 N-1$, and in the system (4.6) there are $2(m-1) N+1$ equations and unknowns.

Having determined the approximation $v_{N}$ for $v=u^{(n)}$, we determine the approximation $J_{0} v_{N}$ for the solution $u=u^{(0)}$ of problem (1.1), (1.2) and the approximations $J_{1} v_{N}, \ldots, J_{n-1} v_{N}$ for the corresponding derivatives $u^{(1)}, \ldots, u^{(n-1)}$ of $u$ with $J_{i}, i=0, \ldots, n-1$, given by $(2.5)$.

For $u^{(n)}$ a better approximation than $v_{N}$ is an iterated approximation of the form (see [2])

$$
\hat{v}_{N}=T_{N} v_{N}+f
$$

with $T_{N}$, defined by the formula (4.1). From (4.4) and (4.7) we obtain that $\mathcal{P}_{N} \hat{v}_{N}=v_{N}$ and therefore

$$
\hat{v}_{N}\left(t_{j k}\right)=v_{N}\left(t_{j k}\right), \quad k=1, \ldots, m, j=1, \ldots, 2 N .
$$

Substituting $v_{N}=\mathcal{P}_{N} \hat{v}_{N}$ into (4.7), we see that $\hat{v}_{N}$ satisfies the equation

$$
\hat{v}_{N}=T_{N} \mathcal{P}_{N} \hat{v}_{N}+f .
$$

\section{Convergence Analysis}

In the sequel, by $c$ and $c_{1}$ we will denote positive constants that are independent of $N$ and may have different values in different occurrences. For given Banach spaces $X$ and $Y$ we denote by $\mathcal{L}(X, Y)$ the Banach space of linear bounded operators $A: X \rightarrow Y$ with the norm

$$
\|A\|_{\mathcal{L}(X, Y)}=\sup \left\{\|A u\|_{Y}: u \in X,\|u\|_{X} \leq 1\right\} .
$$

With Theorem 1 below some general results on the order of convergence of the discrete collocation method are given. 
Theorem 1. Let the conditions of Lemma 2 with $q=m$ be fulfilled, $\mathcal{Q}_{N}=\mathcal{P}_{N}$ and let the nodes (3.2) with grid points (3.1) be used. Then there exists an integer $N_{0} \in \mathbb{N}$ such that, for $N \geq N_{0}$, the equation (4.4) possesses a unique solution $v_{N} \in S_{m-1}^{(-1)}\left(\Pi_{N}\right)$ and the following error estimates hold:

$$
\begin{aligned}
\left\|u^{(i)}-J_{i} v_{N}\right\|_{\infty} & \leq c E_{N}(m, \nu, r), \quad i=0, \ldots, n, \\
\left\|u^{(n)}-\hat{v}_{N}\right\|_{\infty} & \leq c E_{N}(m, \nu, r) .
\end{aligned}
$$

If $n_{0} \leq n-1$ then somewhat more exact estimates are valid:

$$
\begin{aligned}
\left\|u^{(i)}-J_{i} v_{N}\right\|_{\infty} & \leq c \Theta_{N}(m, \nu, r), \quad i=0, \ldots, n-1, \\
\left\|u^{(n)}-\hat{v}_{N}\right\|_{\infty} & \leq c \Theta_{N}(m, \nu, r) .
\end{aligned}
$$

Here $u=u^{(0)}$ is the solution of problem (1.1), (1.2) and $J_{i}, E_{N}, \Theta_{N}$ and $\hat{v}_{N}$ are defined by the formulas (2.5), (3.8), (3.9) and (4.7), respectively.

Proof. First of all we estimate the norm $\left\|T v-T_{N} v\right\|_{\infty}$ for arbitrary $v \in$ $C^{m, \nu}(0, b)$. From $(2.7)$ and $(4.1)$ we get

$$
T v-T_{N} v=\sum_{i=0}^{n_{0}}\left(\tilde{T}_{i}-\tilde{T}_{i N}\right) J_{i} v
$$

Using (2.8) and (4.2) we obtain that

$$
\left(\left(\tilde{T}_{i}-\tilde{T}_{i N}\right) J_{i} v\right)(t)=\int_{0}^{b} g_{i}(t, s)\left(z_{i t}-\mathcal{Q}_{N} z_{i t}\right)(s) d s
$$

where $\mathcal{Q}_{N}=\mathcal{P}_{N}$ and

$$
z_{i t}(s)=K_{i}(t, s)\left(J_{i} v\right)(s), \quad i=0, \ldots, n_{0}, s \in[0, b], t \in[0, b] .
$$

Due to Lemma $1, J_{i} v \in C^{m+n-i, \nu-n+i}(0, b), i=0, \ldots, n_{0}$. This together with $K_{i} \in C^{m}([0, b] \times[0, b])$ yields that (see $\left.(2.1)\right)$

$$
z_{i t} \in C^{m, \nu-n+i}(0, b), \quad i=0, \ldots, n_{0}, t \in[0, b]
$$

and from (3.6) it follows that

$$
\begin{aligned}
\left\|z_{i t}-\mathcal{Q}_{N} z_{i t}\right\|_{\infty} & \leq c E_{N}(m, \nu-n+i, r)\left\|z_{i t}\right\|_{m . \nu-n+i} \\
& \leq c_{1} E_{N}(m, \nu-n+i, r)\|v\|_{m . \nu}, \quad i=0, \ldots, n_{0}
\end{aligned}
$$

where $c_{1}$ does not depend on $t \in[0, b]$. With the help of (1.3) and (5.6) we now obtain that

$$
\begin{aligned}
\sup _{0<t<b}\left|\left(\left(\tilde{T}_{i}-\tilde{T}_{i N}\right) J_{i} v\right)(t)\right| & \leq c \sup _{0<t<b}\left\|z_{i t}-\mathcal{Q}_{N} z_{i t}\right\|_{\infty} \\
& \leq c_{1} E_{N}(m, \nu-n+i, r)\|v\|_{m . \nu}, \quad i=0, \ldots, n_{0} .
\end{aligned}
$$


Therefore we get (see (5.5))

$$
\left\|T v-T_{N} v\right\|_{\infty} \leq c \begin{cases}E_{N}(m, \nu, r)\|v\|_{m . \nu} & \text { if } n_{0}=n \\ \Theta_{N}(m, \nu, r)\|v\|_{m . \nu} & \text { if } 0 \leq n_{0} \leq n-1\end{cases}
$$

Since $C^{m, \nu}(0, b)$ is everywhere dense in $C[0, b]$ and $\left\|T_{N}\right\|_{\mathcal{L}(C[0, b], C[0, b])} \leq c$, we obtain from (3.8), (3.9) and (5.8) that

$$
\left\|T v-T_{N} v\right\|_{\infty} \rightarrow 0 \text { for every } v \in C[0, b] \text { as } N \rightarrow \infty .
$$

Next we observe that $T_{N} \mathcal{P}_{N} \rightarrow T$ compactly in $C[0, b]$ as $N \rightarrow \infty$, i.e.

$$
\left\|T v-T_{N} \mathcal{P}_{N} v\right\|_{\infty} \rightarrow 0 \text { for every } v \in C[0, b] \text { as } N \rightarrow \infty
$$

and for any bounded sequence $\left\{v_{N}\right\}, v_{N} \in C[0, b], N=1,2, \ldots$, it follows that the sequence $\left\{T_{N} \mathcal{P}_{N} v_{N}\right\}$ is relatively compact in $C[0, b]$.

Indeed, we have $\left\|\mathcal{P}_{N}\right\|_{\mathcal{L}\left(C[0, b], L^{\infty}(0, b)\right)} \leq c$ and $\left\|v-\mathcal{P}_{N} v\right\|_{\infty} \rightarrow 0$ for every $v \in C[0, b]$ as $N \rightarrow \infty$ (see $[10,16])$. Further,

$$
T v-T_{N} \mathcal{P}_{N} v=T v-T_{N} v+T_{N}\left(v-\mathcal{P}_{N} v\right) .
$$

This together with (5.9) and $\left\|T_{N}\right\|_{\mathcal{L}\left(C^{(-1)}\left(\Pi_{N}\right), C[0, b]\right)} \leq c$ yields the convergence (5.10). The proof of the relative compactness of the sequence $\left\{T_{N} \mathcal{P}_{N} v_{N}\right\}$ with $\left\|v_{N}\right\|_{\infty} \leq c$ can be built using Lemma 1 and Arzelà theorem (cf. $[2,7]$ ). Since $T$ is compact in $C[0, b]$, the homogeneous equation $v=T v$ has in $C[0, b]$ only the trivial solution $v=0$ and $T_{N} \mathcal{P}_{N} \rightarrow T$ compactly in $C[0, b]$, there exists (see $[2,16]$ ) a number $N_{0} \in \mathbb{N}$ such that, for $N \geq N_{0}$, the operator $\left(I-T_{N} \mathcal{P}_{N}\right)$ is invertible in $C[0, b]$ and

$$
\left\|\left(I-T_{N} \mathcal{P}_{N}\right)^{-1}\right\|_{\mathcal{L}(C[0, b], C[0, b])} \leq c, \quad N \geq N_{0} .
$$

Thus, since $f \in C[0, b]$, the equation (4.8) possesses a unique solution $\hat{v}_{N} \in$ $C[0, b]$ for $N \geq N_{0}$. Consequently, also the equation (4.4) has, for $N \geq N_{0}$, the unique solution $v_{N}=\mathcal{P}_{N} \hat{v}_{N} \in S_{m-1}^{(-1)}\left(\Pi_{N}\right)$.

It follows from (2.6) and (4.8) that

$$
\left(I-T_{N} \mathcal{P}_{N}\right)\left(v-\hat{v}_{N}\right)=T v-T_{N} \mathcal{P}_{N} v
$$

where $v$ and $\hat{v}_{N}$ are the solutions of (2.6) and (4.8), respectively. On the basis of (5.11) and (5.12) we obtain from this that

$$
\left\|v-\hat{v}_{N}\right\|_{\infty} \leq c\left(\left\|T v-T_{N} v\right\|_{\infty}+\left\|T_{N}\left(v-\mathcal{P}_{N} v\right)\right\|_{\infty}\right), \quad N \geq N_{0} .
$$

Using (4.1) we get

$$
\left\|T_{N}\left(v-\mathcal{P}_{N} v\right)\right\|_{\infty} \leq c \sum_{i=0}^{n_{2}}\left\|J_{i}\left(v-\mathcal{P}_{N} v\right)\right\|_{\infty}
$$

where $n_{2}=\max \left\{n_{0}, n_{1}\right\}$. From (2.5) it follows that

$$
\left\|J_{i}\left(v-\mathcal{P}_{N} v\right)\right\|_{\infty} \leq c\left\|v-\mathcal{P}_{N} v\right\|_{1}, \quad i=0, \ldots, n-1 .
$$


Due to Lemma $2, v=u^{(n)} \in C^{m, \nu}(0, b)$. Now (5.13), (5.8), (5.14), (5.15), (3.6) and (3.7) yield the estimates (5.2) and (5.4).

Finally, since $v=u^{(n)} \in C^{m, \nu}(0, b), v_{N}=\mathcal{P}_{N} \hat{v}_{N}$,

$$
\begin{aligned}
& v-v_{N}=\left(v-\mathcal{P}_{N} v\right)+\mathcal{P}_{N}\left(v-\hat{v}_{N}\right), \\
& J_{i}\left(v-v_{N}\right)=J_{i}\left(v-\mathcal{P}_{N} v\right)+J_{i} \mathcal{P}_{N}\left(v-\hat{v}_{N}\right), \quad i=0, \ldots, n,
\end{aligned}
$$

the estimates (5.1) and (5.3) follow from (2.4), (5.2), (5.4), (5.15), (5.16) and Lemma 3.

Remark 4. In [10] in case $0 \leq n_{0} \leq n-1$ for solving (1.1), (1.2) the (exact) collocation method $v_{N}=\mathcal{P}_{N} T v_{N}+\mathcal{P}_{N} f$ has been used. It follows from Theorem 1 that discrete collocation method $v_{N}=\mathcal{P}_{N} T_{N} v_{N}+\mathcal{P}_{N} f$ converges with the same rate as it is proved for the exact collocation method in [10].

\section{$6 \quad$ Higher Order Estimates}

It follows from Theorem 1 that for method (4.4) for every choice of collocation parameters $0 \leq \eta_{1}<\ldots<\eta_{m} \leq 1$ a convergence of order $O\left(N^{-m}\right)$ can be expected, using sufficiently large values of the grid parameter $r$. In this section we show that assuming a little more regularity of functions $f, a_{i}, g_{i}$, $K_{i}, i=0, \ldots, n_{0}$, by a careful choice of parameters $\eta_{1}, \ldots, \eta_{m}$ and $\xi_{1}, \ldots, \xi_{m_{1}}$ in (3.2) and (3.3) it is possible to improve the convergence rate of the discrete collocation method (4.4). For this we will estimate more accurately the norms $\left\|J_{i}\left(v-\mathcal{P}_{N} v\right)\right\|_{\infty}(i=0, \ldots, n-1)$ and $\left\|T v-T_{N} v\right\|_{\infty}$ by using the following Lemmas 4 and 5 , respectively.

Lemma 4. Let $v \in C^{q_{i}, \nu}(0, b)$, where $-\infty<\nu<1$ and $q_{i}=m+\min \{m, n-i\}$ with $m, n \in \mathbb{N}, i \in\{0, \ldots, n-1\}$. Moreover, assume that from all polynomials $u$ of degree $n-1$ only $u=0$ satisfies the conditions (1.2), the nodes (3.2) with grid points (3.1) and parameters $\eta_{1}, \ldots, \eta_{m}$ are used and the quadrature approximation

$$
\int_{0}^{1} F(x) d x \approx \sum_{k=1}^{m} w_{k} F\left(\eta_{k}\right), \quad 0 \leq \eta_{1}<\ldots<\eta_{m} \leq 1,
$$

with appropriate weights $w_{k}=w_{k}^{(m)}(k=1, \ldots, m)$, is exact for all polynomials $F$ of degree $q_{i}-1$. Then for $r \geq 1$ the following estimate holds:

$$
\left\|J_{i}\left(v-\mathcal{P}_{N} v\right)\right\|_{\infty} \leq c \Theta_{N}\left(q_{i}, \nu, r\right) .
$$

Here $c$ is a positive constant not depending on $N$, and $J_{i}, \mathcal{P}_{N}$ and $\Theta_{N}$ are defined by (2.5), (3.4) and (3.9), respectively.

Proof. Suppose that $v \in C^{q_{i}, \nu}(0, b)$. Then

$$
\begin{aligned}
{\left[J_{i}(v\right.} & \left.\left.-\mathcal{P}_{N} v\right)\right](t)=\int_{0}^{b}\left[\frac{\partial^{i} G(t, s)}{\partial t^{i}}-\varphi(s)\right]\left(v-\mathcal{P}_{N} v\right)(s) d s \\
& +\int_{0}^{b} \varphi(s)\left(v-\mathcal{P}_{N} v\right)(s) d s, \quad t \in(0, b), i \in\{0, \ldots, n-1\}
\end{aligned}
$$


where $G(t, s)$ is the Green function of problem (2.2), (1.2) and the function $\varphi(s)$ is generated in following way.

Let us fix $t \in(0, b)$ and $i \in\{0, \ldots, n-1\}$. We denote

$$
\gamma(s)=\frac{\partial^{i} G(t, s)}{\partial t^{i}}, \quad p=\min \{m, n-i\} .
$$

Note that, for simplicity of presentation, we do not show the dependence of $\gamma$ (below also $\varphi$ ) on $t$ and $i$. Let $t \in\left(t_{l-1}, t_{l}\right]$ for an $l \in\{1, \ldots, 2 N\}$. Due to properties of the Green's function $G(t, s)$ we have $\gamma \in C^{p-2}\left[t_{l-1}, t_{l}\right], \gamma^{(p-1)} \in$ $L^{\infty}\left(t_{l-1}, t_{l}\right)$ and $\gamma \in C^{p+1}\left[t_{j-1}, t_{j}\right]$ for $j \neq l, j=1, \ldots, 2 N$. (If $t_{l}=t$ then by $\gamma^{(\mu)}\left(t_{l}\right)$ we denote the right $\left.\operatorname{limit}_{\lim _{s \rightarrow t_{l}, s>t_{l}}} \gamma^{(\mu)}(s), \mu=0, \ldots, p+1\right)$. We define

$$
\varphi(s)= \begin{cases}\sum_{\mu=0}^{p-2} \frac{1}{\mu !} \gamma^{(\mu)}\left(t_{l-1}\right)\left(s-t_{l-1}\right)^{\mu}, & s \in\left(t_{l-1}, t_{l}\right), \\ \sum_{\mu=0}^{p} \frac{1}{\mu !} \gamma^{(\mu)}\left(t_{j-1}\right)\left(s-t_{j-1}\right)^{\mu}, & s \in\left(t_{j-1}, t_{j}\right), j \neq l, j=1, \ldots, 2 N .\end{cases}
$$

If $p=1$ then we take $\varphi(s)=0$ for $s \in\left(t_{l-1}, t_{l}\right)$. Then $\varphi \in S_{p}^{(-1)}\left(\Pi_{N}\right)$ and by using the well known estimates for Taylor expansion we obtain that

$$
\begin{aligned}
& \sup _{t_{l-1}<s<t_{l}}|\gamma(s)-\varphi(s)| \leq c\left(t_{l}-t_{l-1}\right)^{p-1}, \\
& \sup _{t_{j-1}<s<t_{j}}|\gamma(s)-\varphi(s)| \leq c\left(t_{j}-t_{j-1}\right)^{p+1}, \quad j \neq l, j=1, \ldots, 2 N,
\end{aligned}
$$

where $c$ does not depend on $t$. From these estimates and Lemma 3.3 of [10] it follows that (cf. also [16])

$$
\begin{aligned}
& \left|\sum_{j=1, j \neq l}^{2 N} \int_{t_{j-1}}^{t_{j}}\left[\frac{\partial^{i} G(t, s)}{\partial t^{i}}-\varphi(s)\right]\left(v-\mathcal{P}_{N} v\right)(s) d s\right| \leq c N^{-p}\left\|v-\mathcal{P}_{N} v\right\|_{1}, \\
& \left|\int_{t_{l-1}}^{t_{l}}\left[\frac{\partial^{i} G(t, s)}{\partial t^{i}}-\varphi(s)\right]\left(v-\mathcal{P}_{N} v\right)(s) d s\right| \leq c\left(t_{l}-t_{l-1}\right)^{p} \sup _{t_{l-1}<s<t_{l}}\left|\left(v-\mathcal{P}_{N} v\right)(s)\right| \\
& \leq c_{1} \begin{cases}N^{-q_{i}} & \text { if } m<1-\nu, \\
N^{-q_{i}}(1+\log N) & \text { if } m=1-\nu \text { and } r=1, \\
N^{-q_{i}} & \text { if } m=1-\nu \text { and } r>1, \\
N^{-r(p+1-\nu)} & \text { if } m>1-\nu \text { and } 1 \leq r<\frac{q_{i}}{p+1-\nu}, \\
N^{-q_{i}} & \text { if } m>1-\nu \text { and } r \geq \frac{q_{i}}{p+1-\nu} .\end{cases}
\end{aligned}
$$

The last estimates together with (3.7) yield the estimate

$$
\sup _{0<t<b}\left|\int_{0}^{b}\left[\frac{\partial^{i} G(t, s)}{\partial t^{i}}-\varphi(s)\right]\left(v-\mathcal{P}_{N} v\right)(s) d s\right| \leq c \Theta_{N}\left(q_{i}, \nu, r\right) .
$$

Since $\varphi \mathcal{P}_{N} v \in S_{q_{i}-1}^{(-1)}\left(\Pi_{N}\right),\left(\mathcal{P}_{N} v\right)\left(t_{j k}\right)=v\left(t_{j k}\right), k=1, \ldots, m, j=1, \ldots, 2 N$, and the quadrature approximation $(6.1)$ is exact for all polynomials of degree 
$q_{i}-1$, we may write the last integral in $(6.3)$ in the form

$$
\int_{0}^{b} \varphi(s)\left(v-\mathcal{P}_{N} v\right)(s) d s=\sum_{j=1}^{2 N}\left[\int_{t_{j-1}}^{t_{j}} v(s) \varphi(s) d s-\left(t_{j}-t_{j-1}\right) \sum_{k=1}^{m} w_{k} v\left(t_{j k}\right) \varphi\left(t_{j k}\right)\right] .
$$

Estimating the right hand side of the last equality in a similar way as in the proof of Lemma 4.2 of [11] we obtain that

$$
\sup _{0<t<b}\left|\int_{0}^{b} \varphi(s)\left(v-\mathcal{P}_{N} v\right)(s) d s\right| \leq c \Theta_{N}\left(q_{i}, \nu, r\right) .
$$

This together with (6.3) and (6.4) yields the estimate (6.2).

Lemma 5. Assume that $z \in C^{m_{1}+1, \nu_{1}}(0, b)$ and $g \in W^{1, \nu}(\Delta)$ where $m_{1} \in \mathbb{N}$, $-\infty<\nu_{1}<1,-\infty<\nu<1$. Let the interpolation operator $\mathcal{Q}_{N}$ be determined by (3.5) and let the nodes (3.3) with grid points (3.1) and parameters $\xi_{1}, \ldots, \xi_{m_{1}}$ be used. Furthermore, let the quadrature approximation

$$
\int_{0}^{1} F(x) d x \approx \sum_{k=1}^{m_{1}} w_{k} F\left(\xi_{k}\right), \quad 0 \leq \xi_{1}<\ldots<\xi_{m_{1}} \leq 1
$$

with appropriate weights $w_{k}=w_{k}^{\left(m_{1}\right)}\left(k=1, \ldots, m_{1}\right)$, be exact for all polynomials $F$ of degree $m_{1}$. Then

$$
\sup _{0<t<b}\left|\int_{0}^{b} g(t, s)\left(z-\mathcal{Q}_{N} z\right)(s) d s\right| \leq c E_{N}\left(m_{1}, \nu_{1}, r\right) \tau_{N}(\nu)
$$

where $c$ is a positive constant not depending on $N$,

$$
\tau_{N}(\nu)= \begin{cases}N^{-1} & \text { if } \nu<0 \\ N^{-1}(1+\log N) & \text { if } \nu=0 \\ N^{-1+\nu} & \text { if } \nu>0\end{cases}
$$

and $E_{N}$ is defined by (3.8).

Proof. Suppose $z \in C^{m_{1}+1, \nu_{1}}(0, b), g \in W^{1, \nu}(\Delta), m_{1} \in \mathbb{N}, \nu_{1}<1, \nu<1$. For given $t \in(0, b)$ we introduce two sets of indices

$$
\begin{aligned}
& I_{1}(t)=\left\{j:\left[t_{j-1}, t_{j}\right] \cap[t-h, t+h] \neq \emptyset, j=1, \ldots, 2 N\right\}, \\
& I_{2}(t)=\left\{j:\left[t_{j-1}, t_{j}\right] \cap[t-h, t+h]=\emptyset, j=1, \ldots, 2 N\right\},
\end{aligned}
$$

where $h=r b / N$ and $\emptyset$ is the empty set. Then

$$
\int_{0}^{b} g(t, s)\left(z-\mathcal{Q}_{N} z\right)(s) d s=\Sigma_{1}(t)+\Sigma_{2}(t), \quad t \in(0, b),
$$

where

$$
\Sigma_{i}(t)=\sum_{j \in I_{i}(t)} \int_{t_{j-1}}^{t_{j}} g(t, s)\left(z-\mathcal{Q}_{N} z\right)(s) d s, \quad i=1,2
$$


Since $0<t_{j}-t_{j-1} \leq h / 2, j=1, \ldots, 2 N$, we have

$$
\left|\Sigma_{1}(t)\right| \leq\left\|z-\mathcal{Q}_{N} z\right\|_{\infty} \int_{b_{1}}^{b_{2}}|g(t, s)| d s
$$

where $b_{1}=\max \{0, t-3 h / 2\}, b_{2}=\min \{b, t+3 h / 2\}$ and $0<t<b$. This together with (1.3) and (3.6) (where in the role $\mathcal{P}_{N}$ is $\mathcal{Q}_{N}$ ) yields that

$$
\sup _{0<t<b}\left|\Sigma_{1}(t)\right| \leq c E_{N}\left(m_{1}, \nu_{1}, r\right) \tau_{N}(\nu) .
$$

In order to estimate the sum $\Sigma_{2}(t)$, we first add to the parameters $\xi_{1}, \ldots, \xi_{m_{1}}$ a parameter $\xi_{m_{1}+1} \in[0,1]$, different from $\xi_{1}, \ldots, \xi_{m_{1}}$, and, in analogy to $\mathcal{Q}_{N}$, determine the interpolation operator $\mathcal{Q}_{N}^{(1)}$ from the following conditions:

$\mathcal{Q}_{N}^{(1)} v \in S_{m_{1}}^{(-1)}\left(\Pi_{N}\right),\left(\mathcal{Q}_{N}^{(1)} v\right)\left(s_{j k}\right)=v\left(s_{j k}\right), \quad k=1, \ldots, m_{1}+1, j=1, \ldots, 2 N$.

Here $v \in C[0, b]$ and

$$
s_{j k}=t_{j-1}+\xi_{k}\left(t_{j}-t_{j-1}\right), \quad k=1, \ldots, m_{1}+1, j=1, \ldots, 2 N .
$$

Since (6.5) is exact for all polynomials of degree $m_{1}$, we have

$$
\int_{t_{j-1}}^{t_{j}}\left(\mathcal{Q}_{N} z\right)(s) d s=\int_{t_{j-1}}^{t_{j}}\left(\mathcal{Q}_{N}^{(1)} z\right)(s) d s, \quad j=1, \ldots, 2 N .
$$

Using this we obtain that

$$
\Sigma_{2}(t)=\Sigma_{21}(t)+\Sigma_{22}(t), \quad t \in(0, b),
$$

where

$$
\begin{aligned}
& \Sigma_{21}(t)=\sum_{j \in I_{2}(t)} \int_{t_{j-1}}^{t_{j}}\left[g(t, s)-g\left(t, s_{j}\right)\right]\left(z-\mathcal{Q}_{N} z\right)(s) d s, \\
& \Sigma_{22}(t)=\sum_{j \in I_{2}(t)} g\left(t, s_{j}\right) \int_{t_{j-1}}^{t_{j}}\left(z-\mathcal{Q}_{N}^{(1)} z\right)(s) d s
\end{aligned}
$$

and $s_{j}=\left(t_{j-1}+t_{j}\right) / 2, j=1, \ldots, 2 N$. Clearly,

$$
\left|\Sigma_{22}(t)\right| \leq \max _{j \in I_{2}(t)}\left|g\left(t, s_{j}\right)\right|\left\|z-\mathcal{Q}_{N}^{(1)} z\right\|_{1}, \quad t \in(0, b) .
$$

Because $\left|t-s_{j}\right|>h=r b / N$ for $j \in I_{2}(t), t \in(0, b)$, the last estimate together with (1.3) and (3.7) yields that

$$
\sup _{0<t<b}\left|\Sigma_{22}(t)\right| \leq c N \Theta_{N}\left(m_{1}+1, \nu_{1}, r\right) \tau_{N}(\nu) \leq c E_{N}\left(m_{1}, \nu_{1}, r\right) \tau_{N}(\nu) .
$$

An estimate for $\Sigma_{21}(t)$ in (6.10) we obtain in following way. Since $g \in$ $W^{1, \nu}(\Delta)$ and

$$
\frac{\partial g(t, s)}{\partial s}=\left(\frac{\partial}{\partial t}+\frac{\partial}{\partial s}\right) g(t, s)-\frac{\partial}{\partial t} g(t, s)
$$


we get from (1.3) the estimate

$$
\left|\frac{\partial g(t, s)}{\partial s}\right| \leq c H(|t-s|, \nu), \quad(t, s) \in \Delta,
$$

where

$$
H(|t-s|, \nu)= \begin{cases}1 & \text { if } \nu+1<0 \\ 1+|\log | t-s|| & \text { if } \nu+1=0 \\ |t-s|^{-\nu-1} & \text { if } \nu+1>0\end{cases}
$$

If $t \in(0, b), j \in I_{2}(t), s, \sigma \in\left[t_{j-1}, t_{j}\right]$, then

$$
|t-s| \geq h, \quad|t-\sigma| \geq h, \quad \frac{2}{3}|t-s| \leq|t-\sigma| \leq \frac{3}{2}|t-s|,
$$

and consequently,

$$
\left|\frac{\partial g(t, \sigma)}{\partial \sigma}\right| \leq c H(|t-\sigma|, \nu) \leq c_{1} H(|t-s|, \nu) .
$$

Thus, we have for any $t \in(0, b)$ that

$$
\begin{aligned}
\left|\Sigma_{21}(t)\right| & \leq\left\|z-\mathcal{Q}_{N} z\right\|_{\infty} \sum_{j \in I_{2}(t)} \int_{t_{j-1}}^{t_{j}}\left|\int_{s_{j}}^{s} \frac{\partial g(t, \sigma)}{\partial \sigma} d \sigma\right| d s \\
& \leq c E_{N}\left(m_{1}, \nu_{1}, r\right) h \int_{(0, b) \backslash[t-h, t+h]} H(|t-s|, \nu) d s \\
& \leq c_{1} E_{N}\left(m_{1}, \nu_{1}, r\right) \tau_{N}(\nu) .
\end{aligned}
$$

This together with (6.8)-(6.11) yields the estimate (6.6).

Theorem 2. Assume that the following conditions are fulfilled:

(1) the problem (1.1), (1.2) with $f=0$ has only the trivial solution $u=0$ and from all polynomials $u$ of degree $n-1$ only $u=0$ satisfies the conditions (1.2);

(2) $f \in C^{q_{0}, \nu}(0, b), a_{i} \in C^{q_{0}, \nu}(0, b), i=0, \ldots, n_{1}, g_{i} \in W^{q_{0}, \nu}(\Delta), K_{i} \in$ $C^{q_{0}+1}([0, b] \times[0, b]), i=0, \ldots, n_{0}, 0 \leq n_{0} \leq n-1,0 \leq n_{1} \leq n-1$, $q_{0}=m+\min \{m, n\}, m, n \in \mathbb{N},-\infty<\nu<1$;

(3) the interpolation operator $\mathcal{P}_{N}$ is defined by (3.4) where the nodes (3.2) with grid points (3.1) and parameters $0 \leq \eta_{1}<\ldots<\eta_{m} \leq 1$ are used, and the quadrature approximation (6.1) is exact for all polynomials of degree $q_{0}-1$;

(4) the interpolation operator $\mathcal{Q}_{N}$ is defined by (3.5) where the nodes (3.3) with grid points (3.1) and parameters $0 \leq \xi_{1}<\ldots<\xi_{m_{1}} \leq 1$ are used, and the quadrature approximation (6.5) is exact for all polynomials of degree $m_{1}$ where $1 \leq m_{1} \leq q_{0}$. 
Then there exists an integer $N_{0} \in \mathbb{N}$ such that, for $N \geq N_{0}$, the equation (4.4) possesses a unique solution $v_{N} \in S_{m-1}^{(-1)}\left(\Pi_{N}\right)$ and for $r \geq 1$ the following error estimates hold:

$$
\begin{aligned}
& \left\|u^{(i)}-J_{i} v_{N}\right\|_{\infty} \leq c\left[E_{N}\left(m_{1}, \nu-n+n_{0}, r\right) \tau_{N}(\nu)+\Theta_{N}\left(q_{n_{2}}, \nu, r\right)\right], \\
& \quad i=0, \ldots, n_{2}, \\
& \left\|u^{(i)}-J_{i} v_{N}\right\|_{\infty} \leq c\left[E_{N}\left(m_{1}, \nu-n+n_{0}, r\right) \tau_{N}(\nu)+\Theta_{N}\left(q_{i}, \nu, r\right)\right] \\
& i=n_{2}, \ldots, n-1 \\
& \left\|u^{(n)}-\hat{v}_{N}\right\|_{\infty} \leq c\left[E_{N}\left(m_{1}, \nu-n+n_{0}, r\right) \tau_{N}(\nu)+\Theta_{N}\left(q_{n_{2}}, \nu, r\right)\right] .
\end{aligned}
$$

Here $c$ does not depend on $N, n_{2}=\max \left\{n_{0}, n_{1}\right\}, q_{i}=m+\min \{m, n-i\}$, $i=0, \ldots, n-1, u=u^{(0)}$ is the solution of (1.1), (1.2) and $J_{i}, E_{N}, \Theta_{N}, \hat{v}_{N}$ and $\tau_{N}$ are defined by (2.5), (3.8), (3.9), (4.7) and (6.7), respectively.

Proof. In a similar way as in proof of Theorem 1 we get that there exist a number $N_{0} \in \mathbb{N}$ such that, for $N \geq N_{0}$, the equation (4.4) possesses a unique solution $v_{N} \in S_{m-1}^{(-1)}\left(\Pi_{N}\right)$ and the estimates (5.12) and (5.13) hold. Due to Lemma $2 v=u^{(n)} \in C^{q_{0}, \nu}(0, b)$ and therefore $J_{i} v \in C^{q_{0}+n-i, \nu-n+i}(0, b)$, $i=0, \ldots, n-1$ (see Lemma 1$)$. Since $m_{1}+1 \leq q_{0}+1 \leq q_{0}+n-i, i=0, \ldots, n-1$, we obtain that

$$
z_{i t} \in C^{m_{1}+1, \nu-n+i}(0, b), \quad i=0, \ldots, n_{0}, t \in[0, b],
$$

with $z_{i t}$ defined by (5.7). This together with (5.5), (5.6) and Lemma 5 yields (cf. the proof of Theorem 1) that

$$
\left\|T v-T_{N} v\right\|_{\infty} \leq c E_{N}\left(m_{1}, \nu-n+n_{0}, r\right) \tau_{N}(\nu) .
$$

Since $v=u^{(n)} \in C^{q_{0}, \nu}(0, b)$, the estimate (6.14) follows from (5.13), (5.14), (6.2) and (6.15), and the estimates (6.12) and (6.13) follow from (2.4), (5.16), (6.2) and (6.14).

Remark 5. Lemma 4 permits to refine and make more exact some results obtained in [10] for the exact collocation method

$$
v_{N}=\mathcal{P}_{N} T v_{N}+\mathcal{P}_{N} f,
$$

where $T$ and $\mathcal{P}_{N}$ are defined by (2.7) and (3.4), respectively.

More precisely, let the assumptions (1)-(3) of Theorem 2 be fulfilled. Then for sufficiently large $N$ the equation (6.16) possesses a unique solution $v_{N} \in$ $S_{m-1}^{(-1)}\left(\Pi_{N}\right)$ and the following error estimates hold:

$$
\begin{aligned}
& \max _{0 \leq j \leq i}\left\|u^{(j)}-J_{j} v_{N}\right\|_{\infty} \leq c \Theta_{N}\left(q_{i}, \nu, r\right), \quad i=n_{2}, \ldots, n-1, \\
& \left\|u^{(n)}-\hat{v}_{N}\right\|_{\infty} \leq c \Theta_{N}\left(q_{n_{2}}, \nu, r\right) .
\end{aligned}
$$

Here $u=u^{(0)}$ is the solution of (1.1), (1.2), $\hat{v}_{N}=T v_{N}+f, n_{2}=\max \left\{n_{0}, n_{1}\right\}$, $q_{i}=m+\min \{m, n-i\}, i=0, \ldots, n-1$, and $J_{j}$ and $\Theta_{N}$ are defined by $(2.5)$ and (3.9), respectively. 


\section{Numerical Experiments}

Let us consider the following boundary value problem:

$$
\begin{aligned}
& u^{\prime \prime}(t)=\sqrt{t} u(t)+\int_{0}^{1}|t-s|^{-1 / 2} u(s) d s+f(t), \quad t \in[0,1], \\
& u(0)=u^{\prime}(1)=0 .
\end{aligned}
$$

The function $f(t)$ is selected so that

$$
u(t)=t^{5 / 2}+(1-t)^{5 / 2}-1-2.5 t
$$

is the exact solution of this problem (see [12]). Actually, this is a problem of the form (1.1), (1.2), where $n=2, n_{0}=n_{1}=0, b=1, a_{0}(t)=\sqrt{t}$, $g_{0}(t, s)=|t-s|^{-1 / 2}$ and $K_{0}(t, s)=1$. It is easy to check that $a_{0}, f \in C^{q, \nu}(0,1)$, $g_{0} \in W^{q, \nu}(\Delta)$ with $\nu=1 / 2$ and arbitrary $q \in \mathbb{N}$.

Problem (7.1), (7.2) is solved numerically by discrete collocation method (4.4) in the case that $m=2$ and $\eta_{1}=(3-\sqrt{3}) / 6, \eta_{2}=1-\eta_{1}$ are the nodes of the Gaussian quadrature formula (6.1) which is exact for all polynomials of degree 3. Having determined $v_{N}$, the approximation $\hat{v}_{N}$ (which below is denoted by $u_{N}^{(2)}$ ) to $u^{(2)}=u^{\prime \prime}$ is evaluated by (4.7), the approximations $u_{N}^{(0)}=$ $J_{0} v_{N}$ to $u^{(0)}=u$ and $u_{N}^{(1)}=J_{1} v_{N}$ to $u^{(1)}=u^{\prime}$ are found by the formula $(2.5)$ where $b=1$ and

$$
G(t, s)= \begin{cases}-t & \text { for } t<s \\ -s & \text { for } t>s\end{cases}
$$

In Tables 1 and 2 some results for different values of $N$ and $r$ are presented. The quantities $\varepsilon_{N}^{(i)}(i=0,1,2)$ in tables are the approximate values of the norms $\left\|u^{(i)}-u_{N}^{(i)}\right\|_{\infty}(i=0,1,2)$ calculated as follows:

$$
\varepsilon_{N}^{(i)}=\max _{j=1, \ldots, 2 N} \max _{k=0, \ldots, 10}\left|u^{(i)}\left(\tau_{j k}\right)-u_{N}^{(i)}\left(\tau_{j k}\right)\right|, \quad i=0,1,2 .
$$

Here, $\tau_{j k}=t_{j-1}+k\left(t_{j}-t_{j-1}\right) / 10, k=0, \ldots, 10, j=1, \ldots, 2 N$, with the grid points $\left\{t_{j}\right\}$, defined by the formula $(3.1)$ for $b=1$. The ratios $\varrho_{N}^{(i)}=\varepsilon_{N / 2}^{(i)} / \varepsilon_{N}^{(i)}$ $(i=0,1,2)$ characterizing the observed convergence rate, are also presented.

Table 1 shows the dependence of the convergence rate on the grid parameter $r$, when the values $m=m_{1}=2$ and Gaussian parameters $\eta_{1}=\xi_{1}=(3-\sqrt{3}) / 6$, $\eta_{2}=\xi_{2}=(3+\sqrt{3}) / 6$ are used. Then it follows from the estimates $(6.13)$ and (6.14) that for sufficiently large $N$

$$
\max _{i=0,1,2} \varepsilon_{N}^{(i)} \approx \max _{i=0,1,2}\left\|u^{(i)}-u_{N}^{(i)}\right\|_{\infty} \leq c \begin{cases}N^{-1.5 r} & \text { if } 1 \leq r<5 / 3 \\ N^{-2.5} & \text { if } r \geq 5 / 3 \approx 1.67 .\end{cases}
$$

Thus, for $r=1$ and $r \geq 1.7$ the values of ratios $\varrho_{N}^{(i)}(i=0,1,2)$ ought to be approximately $2^{1.5} \approx 2.828$ and $2^{2.5} \approx 5.66$, respectively.

The results in Table 2 correspond to the case when $m=2, m_{1}=3$ and the nodes of Gaussian quadrature formulas are used. Then it follows from 
Table 1. Results in the case $m=m_{1}=2, \eta_{1}=\xi_{1}=(3-\sqrt{3}) / 6, \eta_{2}=\xi_{2}=1-\eta_{1}$.

\begin{tabular}{|c|c|c|c|c|c|c|c|c|}
\hline \multirow[b]{2}{*}{$N$} & \multicolumn{2}{|c|}{$r=1$} & \multicolumn{2}{|c|}{$r=1.7$} & \multicolumn{2}{|c|}{$r=2$} & \multicolumn{2}{|c|}{$r=2.4$} \\
\hline & $\varepsilon_{N}^{(0)}$ & $\varrho_{N}^{(0)}$ & $\varepsilon_{N}^{(0)}$ & $\varrho_{N}^{(0)}$ & $\varepsilon_{N}^{(0)}$ & $\varrho_{N}^{(0)}$ & $\varepsilon_{N}^{(0)}$ & $\varrho_{N}^{(0)}$ \\
\hline 4 & $6.6 \mathrm{E}-4$ & 2.90 & $1.6 \mathrm{E}-4$ & 6.05 & $9.5 \mathrm{E}-5$ & 8.6 & $6.8 \mathrm{E}-5$ & 12.3 \\
\hline 8 & $2.3 \mathrm{E}-4$ & 2.85 & $2.6 \mathrm{E}-5$ & 6.00 & $9.7 \mathrm{E}-6$ & 9.7 & $1.2 \mathrm{E}-5$ & 5.9 \\
\hline 16 & $8.2 \mathrm{E}-5$ & 2.83 & $4.4 \mathrm{E}-6$ & 5.95 & $9.2 \mathrm{E}-7$ & 10.6 & $2.1 \mathrm{E}-6$ & 5.4 \\
\hline 32 & $2.9 \mathrm{E}-5$ & 2.83 & $7.4 \mathrm{E}-7$ & 5.93 & $1.9 \mathrm{E}-7$ & 4.9 & $3.9 \mathrm{E}-7$ & 5.5 \\
\hline 64 & $1.0 \mathrm{E}-5$ & 2.83 & $1.3 \mathrm{E}-7$ & 5.92 & $3.7 \mathrm{E}-8$ & 5.0 & $7.2 \mathrm{E}-8$ & 5.5 \\
\hline$N$ & $\varepsilon_{N}^{(1)}$ & $\varrho_{N}^{(1)}$ & $\varepsilon_{N}^{(1)}$ & $\varrho_{N}^{(1)}$ & $\varepsilon_{N}^{(1)}$ & $\varrho_{N}^{(1)}$ & $\varepsilon_{N}^{(1)}$ & $\varrho_{N}^{(1)}$ \\
\hline 4 & $2.6 \mathrm{E}-3$ & 2.83 & $6.5 \mathrm{E}-4$ & 5.85 & $6.4 \mathrm{E}-4$ & 7.2 & $8.8 \mathrm{E}-4$ & 6.7 \\
\hline 8 & $9.2 \mathrm{E}-4$ & 2.82 & $1.1 \mathrm{E}-4$ & 5.75 & $8.5 \mathrm{E}-5$ & 7.5 & $1.2 \mathrm{E}-4$ & 7.3 \\
\hline 16 & $3.3 \mathrm{E}-4$ & 2.82 & $1.9 \mathrm{E}-5$ & 5.82 & $1.1 \mathrm{E}-5$ & 7.9 & $1.8 \mathrm{E}-5$ & 6.9 \\
\hline 32 & $1.2 \mathrm{E}-4$ & 2.82 & $3.3 \mathrm{E}-6$ & 5.84 & $1.3 \mathrm{E}-6$ & 8.2 & $2.4 \mathrm{E}-6$ & 7.2 \\
\hline 64 & $4.1 \mathrm{E}-5$ & 2.83 & $5.7 \mathrm{E}-7$ & 5.85 & $1.8 \mathrm{E}-7$ & 7.3 & $3.3 \mathrm{E}-7$ & 7.3 \\
\hline$N$ & $\varepsilon_{N}^{(2)}$ & $\varrho_{N}^{(2)}$ & $\varepsilon_{N}^{(2)}$ & $\varrho_{N}^{(2)}$ & $\varepsilon_{N}^{(2)}$ & $\varrho_{N}^{(2)}$ & $\varepsilon_{N}^{(2)}$ & $\varrho_{N}^{(2)}$ \\
\hline 4 & $3.2 \mathrm{E}-3$ & 4.36 & $6.0 \mathrm{E}-3$ & 4.27 & $7.9 \mathrm{E}-3$ & 3.9 & $1.1 \mathrm{E}-2$ & 3.4 \\
\hline 8 & $8.2 \mathrm{E}-4$ & 3.83 & $1.2 \mathrm{E}-3$ & 4.99 & $1.7 \mathrm{E}-3$ & 4.8 & $2.4 \mathrm{E}-3$ & 4.4 \\
\hline 16 & $2.4 \mathrm{E}-4$ & 3.41 & $2.2 \mathrm{E}-4$ & 5.33 & $3.2 \mathrm{E}-4$ & 5.2 & $4.9 \mathrm{E}-4$ & 5.0 \\
\hline 32 & $7.6 \mathrm{E}-5$ & 3.15 & $4.1 \mathrm{E}-5$ & 5.49 & $5.9 \mathrm{E}-5$ & 5.4 & $9.1 \mathrm{E}-5$ & 5.3 \\
\hline 64 & $2.5 \mathrm{E}-5$ & 3.00 & $7.3 \mathrm{E}-6$ & 5.58 & $1.1 \mathrm{E}-5$ & 5.5 & $1.7 \mathrm{E}-5$ & 5.5 \\
\hline
\end{tabular}

Table 2. Results in the case $m=2, \eta_{1}=(3-\sqrt{3}) / 6, \eta_{2}=1-\eta_{1}, m_{1}=3, \xi_{1}=$ $(5-\sqrt{15}) / 10, \xi_{2}=1 / 2, \xi_{3}=1-\xi_{1}$.

\begin{tabular}{|c|c|c|c|c|c|c|c|c|}
\hline \multirow[b]{2}{*}{$N$} & \multicolumn{2}{|c|}{$r=1$} & \multicolumn{2}{|c|}{$r=1.7$} & \multicolumn{2}{|c|}{$r=2$} & \multicolumn{2}{|c|}{$r=2.4$} \\
\hline & $\varepsilon_{N}^{(0)}$ & $\varrho_{N}^{(0)}$ & $\varepsilon_{N}^{(0)}$ & $\varrho_{N}^{(0)}$ & $\varepsilon_{N}^{(0)}$ & $\varrho_{N}^{(0)}$ & $\varepsilon_{N}^{(0)}$ & $\varrho_{N}^{(0)}$ \\
\hline 4 & $6.8 \mathrm{E}-4$ & 2.92 & $1.9 \mathrm{E}-4$ & 5.81 & $1.4 \mathrm{E}-4$ & 7.2 & $1.3 \mathrm{E}-4$ & 8.5 \\
\hline 8 & $2.4 \mathrm{E}-4$ & 2.87 & $3.3 \mathrm{E}-5$ & 5.88 & $1.8 \mathrm{E}-5$ & 7.7 & $1.2 \mathrm{E}-5$ & 10.0 \\
\hline 16 & $8.2 \mathrm{E}-5$ & 2.85 & $5.5 \mathrm{E}-6$ & 5.88 & $2.3 \mathrm{E}-6$ & 7.9 & $1.2 \mathrm{E}-6$ & 10.8 \\
\hline 32 & $2.9 \mathrm{E}-5$ & 2.84 & $9.4 \mathrm{E}-7$ & 5.87 & $2.9 \mathrm{E}-7$ & 7.9 & $1.1 \mathrm{E}-7$ & 10.8 \\
\hline 64 & $1.0 \mathrm{E}-5$ & 2.83 & $1.6 \mathrm{E}-7$ & 5.84 & $3.9 \mathrm{E}-8$ & 7.4 & $5.2 \mathrm{E}-8$ & 2.1 \\
\hline$N$ & $\varepsilon_{N}^{(1)}$ & $\varrho_{N}^{(1)}$ & $\varepsilon_{N}^{(1)}$ & $\varrho_{N}^{(1)}$ & $\varepsilon_{N}^{(1)}$ & $\varrho_{N}^{(1)}$ & $\varepsilon_{N}^{(1)}$ & $\varrho_{N}^{(1)}$ \\
\hline 4 & $2.6 \mathrm{E}-3$ & 2.82 & $6.5 \mathrm{E}-4$ & 6.05 & $6.7 \mathrm{E}-4$ & 7.1 & $9.3 \mathrm{E}-4$ & 6.6 \\
\hline 8 & $9.2 \mathrm{E}-4$ & 2.82 & $1.1 \mathrm{E}-4$ & 5.75 & $9.0 \mathrm{E}-5$ & 7.4 & $1.3 \mathrm{E}-4$ & 7.2 \\
\hline 16 & $3.3 \mathrm{E}-4$ & 2.82 & $2.0 \mathrm{E}-5$ & 5.81 & $1.2 \mathrm{E}-5$ & 7.6 & $1.7 \mathrm{E}-5$ & 7.5 \\
\hline 32 & $1.2 \mathrm{E}-4$ & 2.82 & $3.3 \mathrm{E}-6$ & 5.84 & $1.5 \mathrm{E}-6$ & 7.8 & $2.2 \mathrm{E}-6$ & 7.7 \\
\hline 64 & $4.1 \mathrm{E}-5$ & 2.83 & $5.7 \mathrm{E}-7$ & 5.85 & $2.0 \mathrm{E}-7$ & 7.8 & $4.6 \mathrm{E}-7$ & 4.8 \\
\hline$N$ & $\varepsilon_{N}^{(2)}$ & $\varrho_{N}^{(2)}$ & $\varepsilon_{N}^{(2)}$ & $\varrho_{N}^{(2)}$ & $\varepsilon_{N}^{(2)}$ & $\varrho_{N}^{(2)}$ & $\varepsilon_{N}^{(2)}$ & $\varrho_{N}^{(2)}$ \\
\hline 4 & $1.5 \mathrm{E}-3$ & 2.85 & $4.3 \mathrm{E}-4$ & 5.62 & $3.1 \mathrm{E}-4$ & 7.0 & $2.7 \mathrm{E}-4$ & 8.2 \\
\hline 8 & $5.3 \mathrm{E}-4$ & 2.85 & $7.5 \mathrm{E}-5$ & 5.79 & $4.2 \mathrm{E}-5$ & 7.6 & $2.8 \mathrm{E}-5$ & 9.8 \\
\hline 16 & $1.9 \mathrm{E}-4$ & 2.80 & $1.3 \mathrm{E}-5$ & 5.82 & $5.3 \mathrm{E}-6$ & 7.8 & $2.6 \mathrm{E}-6$ & 10.6 \\
\hline 32 & $6.8 \mathrm{E}-5$ & 2.82 & $2.2 \mathrm{E}-6$ & 5.84 & $6.8 \mathrm{E}-7$ & 7.9 & $5.7 \mathrm{E}-7$ & 4.6 \\
\hline 64 & $2.4 \mathrm{E}-5$ & 2.82 & $4.0 \mathrm{E}-7$ & 5.52 & $5.1 \mathrm{E}-7$ & 1.3 & $1.7 \mathrm{E}-5$ & 0.03 \\
\hline
\end{tabular}


Theorem 2 that for sufficiently large $N$ the following error estimates hold:

$$
\begin{aligned}
& \max _{i=0,2} \varepsilon_{N}^{(i)} \approx \max _{i=0,2}\left\|u^{(i)}-u_{N}^{(i)}\right\|_{\infty} \leq c \begin{cases}N^{-1.5 r} & \text { if } 1 \leq r<7 / 3, \\
N^{-3.5} & \text { if } r \geq 7 / 3 \approx 2.33,\end{cases} \\
& \varepsilon_{N}^{(1)} \approx\left\|u^{(1)}-u_{N}^{(1)}\right\|_{\infty} \leq c \begin{cases}N^{-1.5 r} & \text { if } 1 \leq r<2, \\
N^{-3}(1+\log N) & \text { if } r=2, \\
N^{-3} & \text { if } r>2 .\end{cases}
\end{aligned}
$$

We see that for $1 \leq r<1.7$ the method ought to converge with the same speed as in the case of Table 1, but for $r>1.7$ the convergence is faster. For $r=2$ the values $\varrho_{N}^{(i)}(i=0,1,2)$ ought to be approximatively $2^{3}=8$ and for $r=2.4$ the values $\varrho_{N}^{(1)}$ and $\varrho_{N}^{(i)}(i=0,2)$ approximatively 8 and $2^{3.5} \approx 11.3$, respectively.

The presented numerical results show that the error estimates of Theorem 2 are in good agreement with the actual convergence rate of the discrete collocation method. Note that in finding $u_{64}^{(2)}=\hat{v}_{64}$ in the case $r=2$ and $u_{64}^{(0)}=J_{0} v_{64}$, $u_{64}^{(1)}=J_{1} v_{64}, u_{32}^{(2)}=\hat{v}_{32}$ and $u_{64}^{(2)}=\hat{v}_{64}$ in the case $r=2.4$ a numerical instability is observed (see the values $\varrho_{64}^{(0)}, \varrho_{64}^{(1)}, \varrho_{32}^{(2)}$ and $\varrho_{64}^{(2)}$ in Table 2). This effect is discussed in [5].

In [12] for the numerical solution of problem (7.1), (7.2) a fully discrete version of the Galerkin method is used. We see that the discrete collocation method gives for the same $N$ the approximations to the solution nearly with the same accuracy. But the algorithm of the discrete collocation method is more simple.

\section{Acknowledgment}

The authors are very grateful to the referee for his careful reading, valuable comments and helpful suggestions.

\section{References}

[1] C. Allouch, P. Sablonnière, D. Sbibih and M. Tahrichi. Product integration methods based on discrete spline quasi-interpolants and application to weakly singular integral equations. J. Comput. Appl. Math., 233(11):2855-2866, 2010. Doi:10.1016/j.cam.2009.11.031.

[2] K.E. Atkinson. The Numerical Solution of Integral Equations of the Second Kind. Cambridge Monogr. Appl. Comput. Math., 4. Cambridge University Press, Cambridge, UK, 1997.

[3] H. Brunner. Collocation Methods for Volterra Integral and Related Functional Equations. Cambridge Monogr. Appl. Comput. Math., 15. Cambridge University Press, Cambridge, UK, 2004.

[4] Y. Cao, M. Huang, L. Liu and Y. Xu. Hybrid collocation methods for Fredholm integral equations with weakly singular kernels. Appl. Numer. Math., 57(57):549-561, 2007. Doi:10.1016/j.apnum.2006.07.007.

[5] R. Kangro and I. Kangro. On the stability of piecewise polynomial collocation methods for solving weakly singular integral equations of the second kind. Math. Model. Anal., 13(1):29-36, 2008. Doi:10.3846/1392-6292.2008.13.29-36. 
[6] R. Kangro and E. Tamme. On fully discrete collocation methods for solving weakly singular integro-differential equations. Math. Model. Anal., 15(1):69-82, 2010. Doi:10.3846/1392-6292.2010.15.69-82.

[7] K. Orav-Puurand, A. Pedas and G. Vainikko. Nyström type methods for Fredholm integral equations with weak singularities. J. Comput. Appl. Math., 234(9):2848-2858, 2010. Doi:10.1016/j.cam.2010.01.033.

[8] K. Orav-Puurand and G. Vainikko. Central part interpolation schemes for integral equations. Numer. Funct. Anal. Optim., 30(3-4):352-370, 2009. Doi:10.1080/01630560902841153.

[9] I. Parts, A. Pedas and E. Tamme. Piecewise polynomial collocation for Fredholm integro-differential equations with weakly singular kernels. SIAM J. Numer. Anal., 43(5):1897-1911, 2005. Doi:10.1137/040612452.

[10] A. Pedas and E. Tamme. Spline collocation method for integro-differential equations with weakly singular kernels. J. Comput. Appl. Math., 197(1):253-269, 2006. Doi:10.1016/j.cam.2005.07.035.

[11] A. Pedas and E. Tamme. Discrete Galerkin method for Fredholm integrodifferential equations with weakly singular kernels. J. Comput. Appl. Math., 213(1):111-126, 2008. Doi:10.1016/j.cam.2006.12.024.

[12] A. Pedas and E. Tamme. Fully discrete Galerkin method for Fredholm integrodifferential equations with weakly singular kernels. Comput. Methods. Appl. Math., 8(3):294-308, 2008.

[13] A. Pedas and G. Vainikko. On the regularity of solutions to integral equations with nonsmooth kernels on a union of open intervals. J. Comput. Appl. Math., 229(2):440-451, 2009. Doi:10.1016/j.cam.2008.04.009.

[14] C. Schneider. Product integration for weakly singular integral equations. Math. Comp., 36(153):207-213, 1981. Doi:10.1090/S0025-5718-1981-0595053-0.

[15] E. Vainikko and G. Vainikko. A spline quasi-interpolation method for weakly singular Fredholm integral equations. SIAM J. Numer. Anal., 46(4):1799-1820, 2008. Doi:10.1137/070693308.

[16] G. Vainikko. Multidimensional Weakly Singular Integral Equations. Lecture Notes in Math., 1549. Springer-Verlag, 1993. 